ptome, die er darbot, waren die einer Tabes. Der 2. Patient, der vor 23 Jahren Syphilis akquiriert hatte, zeigte spastische Erscheinungen des. rechten Beines, doch erhaltene Kremaster-, Blasen- und Rectumreflexe, weiters Schmerzen in der Lumbalgegend, Symptome, die auf ein Gumma, in der Pia mater der rechten Seite des Rückenmarks hinwiesen, ungefähr am oberen Ende der Lumbalanschwellung des Rückenmarkes.

R. Böhm (Prag).

Scherer. Die Parrotschen Pseudoparalysen bei angeborener Syphilis. Dermatol. Zeitschr. Bd. IX, 1902.

Scherer hat in zwei Fällen von Lähmungen der oberen Extremitäten bei syphilitischen Kindern das Rückenmark untersucht. Die Lähmung trat in einem Falle am 8., im anderen am 5. Tage auf. Die leicht erweiterten Blutgefäße des Cervikalganglion sind erfüllt von Streptokokken-Reinkulturen, die Kapillaren zeigen das Bild einer Streptokokken-Embolie. In der Umgebung keine Reaktion. In den inneren Organen fanden sich ebenfalls reichliche Streptokokken. Zwei intravenőse Injektionen von Streptokokken-Kulturen erzeugten bei Kaninchen Paralyse der hinteren Extremitäten. Verfasser bespricht die in der Literatur bekannten analogen Fälle. Er nimmt für seine Fälle das Streptokokken-Toxin als Ursache der Lähmung an. Fritz Porges (Prag).

\title{
Hereditäre Syphilis.
}

Macitwaine, S. W. Syphilitic Heredity, British Medical Journal 15. Juni 1901.

Ein 47jähriger Mann akquirierte vor ca. 20 Jahren Syphilis, die nicht einer radikalen Kur unterzogen wurde. Sonst war der Mann ein mäßiger Trinker. Bis vor 4 Jahren völlige Gesundheit, jetzt Herzbeschworden und beginnender Hydrops. Vor ca. 12 Jahren Ehe, aus der ein 10jähriger und 8jähriger Knabe stammen. Beide litten früher an Rhachitis. Der ältere Knabe seit zwei Jahren krank, zeigt große Schwäche, geistigen Verfall, Incontinentia urinae, in letzter Zeit auch Incontinentia alvi. Das jüngere Kind leidet seit einem vor $2 \frac{1}{2}$ Jahren erfolgten Sturze an Epilepsie. Verfasser führt sowohl die Herzaffektion des Vaters als auch die Erkrankung der Kinder auf die Infektion mit Syphilis zurück. Verfasser ist der Meinung, dali erst nach einer 2-3jährigen energischen Behandlung und einem mehrjährigen rezidivfreien Intervall die Erlaubnis zur Ehe gegeben werden soll.

R. Böhm (Prag).

\section{Therapie.}

Winckler, A. Über den Nutzen der Kombination von Schmierkur und Schwefelkur bei Behandlang der Syphilis. Deutsche Medizinalzeitung 1902, Nr. 33. 